\title{
Nonlinear Hall effect in the field-induced spin-density wave states of $(\text { TMTSF })_{2} \mathbf{P F}_{6}$
}

\author{
L. BALICAS, N. BIŠKUP ${ }^{(1)}$ and G. KRIZA $(2)$
}

Laboratoire de Physique des Solides, URA 2 CNRS, Université Paris-Sud, 91405 Orsay, France

The resistivity along the conducting chains, $\rho_{x x}$, and the Hall resistivity $\rho_{x y}$ are investigated as a function of the electric field in the magnetic-field-induced spin-density wave (FISDW) states of (TMTSF) ${ }_{2} \mathrm{PF}_{6}$ under hydrostatic pressure. Both resistivities decrease with increasing electric field above the same well-defined threshold electric field in the order of $100 \mu \mathrm{V} / \mathrm{cm}$. We interpret the nonlinearity by the depinning and sliding of the FISDW. The nonlinearity in $\rho_{x y}$ is much stronger than in $\rho_{x x}$ what we attribute to an unusually strong coupling between the FISDW and single-particle excitations.

So far the only bulk systems found to show quantum Hall effect are the Bechgaard salts (TMTSF) ${ }_{2} X$ (TMTSF is tetramethyltetraselenafulvalene, $X=\mathrm{PF}_{6}, \mathrm{ClO}_{4}, \mathrm{ReO}_{4}$, etc.) in the magnetic-field-induced spin-density wave (FISDW) state [1]. The best understood of these systems is (TMTSF) ${ }_{2} \mathrm{PF}_{6}$ where FISDW's are observed above a critical pressure $p_{c} \approx 6 \mathrm{kbar}$. The ground state without magnetic field is superconducting. The metallic state is restored by a magnetic field of about $0.06 \mathrm{~T}$, and by further increasing the field, a cascade of phase transitions to FISDW states occur. These FISDW states are different from the insulating "ordinary" SDW ground state of the same material at ambient pressure, because the FISDW coexists with electrons occupying quasi-2D Landau levels [2]. In each FISDW state a small number $n$ of Landau levels are fully occupied while the others are empty, and, consequently, quantum Hall effect (QHE) is obtained with a Hall conductivity $\sigma_{x y}=2 n e^{2} / h$. When the QHE is observed, the FISDW is pinned to the crystal lattice by defects. On the other hand, the theoretical expectation [3] for the opposite extreme case when the FISDW is free to move along the $1 \mathrm{D}$ chains, is $\sigma_{x y}=\mathbf{0}$ for any finite frequency, i.e., the QHE is expected to disappear. For a more realistic situation, i.e., when the FISDW is depinned from the defects, but its motion is damped by the interaction with other excitations in the system, a finite $\sigma_{x y}$, which is, however, smaller than the QHE value, might be expected.

So far the only experimental test of this problem has been performed on (TMTSF) ${ }_{2} \mathrm{ClO}_{4}$ by Osada et al. [4] Surprisingly, they have found that $\rho_{x x}$ ( $x$ is in the chain direction) increases rather than decreases as a function of the longitudinal electric field $E_{x}$, while the Hall resistivity $\rho_{x y}$ does not change. In conttrast, a decreasing $\rho_{x x}$ has been reported for (TMTSF) ${ }_{2} \mathrm{PF}_{6}$ [5].

In this paper we report on the observation of a nonlinear $\rho_{x x}$ and $\rho_{x y}$ in the FISDW states of (TMTSF) ${ }_{2} \mathrm{PF}_{6}$ : both resistivities decrease with increasing longitudinal field above the same welldefined threshold field $E_{T}$. The magnitude, temperature-, and magnetic-field dependence of $E_{T}$ is in agreement with expectations for FISDW depinning. We attribute the nonlinearity in $\rho_{x x}$ to the charge transport by the sliding FISDW, and interpret the nonlinear $\rho_{x y}$ in terms of an interaction between the condensed (FISDW) and non-condensed ("normal") electrons.

(1)

(2)

On leave from the Institute of Physics, University of Zagreb, Zagreb, Croatia

Also at Research Institute for Solid State Physics, P.O. Box 49, 1525 Budapest, Hungary 


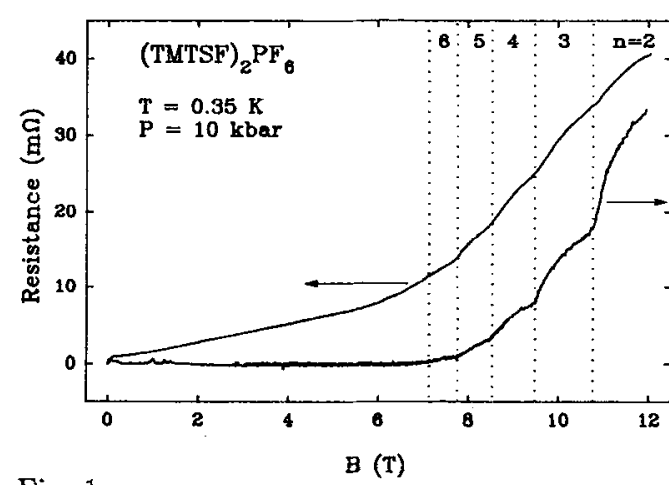

Fig. 1

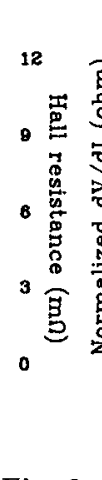

Fig. 2

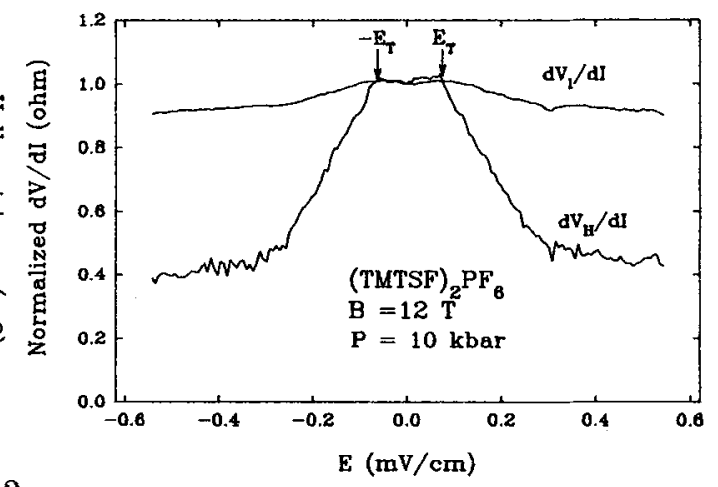

E $(\mathrm{mV} / \mathrm{cm})$

Figure 1: Resistance along the conducting chains and Hall resistance as a function of magnetic field. The pointed lines indicate the transitions to FISDW states with index $n$.

Figure 2: Differential resistance $d V_{l} / d I$ and differential Hall resistance $d V_{H} / d I$ as a function of electric field, normalized to the low-electric-field values. Arrows indicate the threshold field for nonlinearity.

The experiments have been performed in a pressure of $10 \mathrm{kbar}$, and in a magnetic field $B=0$ to $12 \mathrm{~T}$ perpendicular to the quasi-two-dimensional $a-b$ plane at temperatures down to $350 \mathrm{~m} \mathbf{K}$. The differential resistances $d V_{l} / d I$ and $d V_{H} / d I$ are measured, where $I$ is the current along the conducting chains, $V_{l}$ is the longitudinal voltage, and $V_{H}$ is the Hall voltage. To cancel out the admixture of longitudinal and transverse voltages, all the measurements are repeated with positive and negative magnetic fields, and the symmetric and antisymmetric parts are taken for $V_{l}$ and $V_{H}$, respectively. The longitudinal and Hall resistances, $R$ and $R_{H}$, measured this way as a function of the magnetic field $B$ are shown in Fig. 1. Both quantities reflect the cascade of FISDW transitions as indicated in the figure.

The nonlinear transport have been investigated by measuring $d V_{l} / d I$ and $d V_{H} / d I$ using a finite dc bias current $I$ with a small-amplitude low-frequency modulation to obtain the differential resistances. The differential resistances as a function of the longitudinal electric field $E_{x}=V_{l} / l(l$ is the distance of voltage contacts) are shown in Fig. 2. Both quantities start to decrease above a sharp threshold electric field $E_{T}$ in the order of $100 \mu \mathrm{V} / \mathrm{cm}$, and we will argue that the nonlinearity originates from the depinning and sliding of the FISDW's. The threshold field increases both with increasing temperature and magnetic field, as displayed in Figs. 3 and 4, respectively.

Before an analysis of the nonlinear Hall effect, we would like to show that the magnitude, temperature-, and magnetic field dependence of $E_{T}$ is in good agreement with general expectations for density wave depinning. Although the threshold field depends on many details of the system, the general trend (expected from theory and found in experiments) is that with decreasing order parameter $\Delta, E_{T}$ decreases. If the only parameter that changes in the system is $\Delta$, the simplest theory of pinning [6] gives either $E_{T} \propto \Delta$ ("strong pinning limit") or $E_{T} \propto \Delta^{4}$ ("weak pinning limit"). The experimental values lie between these two extremes; for example, $E_{T} \propto \Delta^{2}$ is found for the charge-density wave depinning in $\mathrm{K}_{0.3} \mathrm{MoO}_{3}$ under hydrostatic pressure. [7]

We can use this framework for an order-of-magnitude comparison of the $E_{T}$ found in FISDW's with the SDW depinning threshold of the same material at ambient pressure. In the latter case $\Delta \approx 20 \mathrm{~K}$, the SDW transition temperature is $T_{c}=12 \mathrm{~K}$, and the threshold field is 2 to $4 \mathrm{mV} / \mathrm{cm}$ in the same batch as used in our study of FISDW's. If one assumes that $\Delta / T_{c}$ is the same in the two cases, the $E_{T}^{\mathrm{FISDW}}=70 \mu \mathrm{V} / \mathrm{cm}$ and $T_{c}=1.7 \mathrm{~K}$ values give $E_{T}^{\mathrm{FISDW}} / E_{T}^{\mathrm{SDW}} \approx 40 \approx\left(\Delta^{\mathrm{FISDW}} / \Delta^{\mathrm{SDW}}\right)^{2}$ indicating that the observed $E_{T}$ is in the order of magnitude expected from the low transition temperature of the 


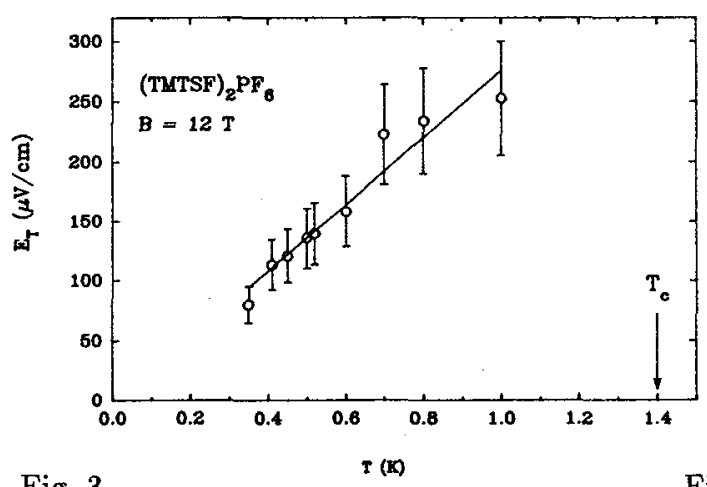

Fig. 3

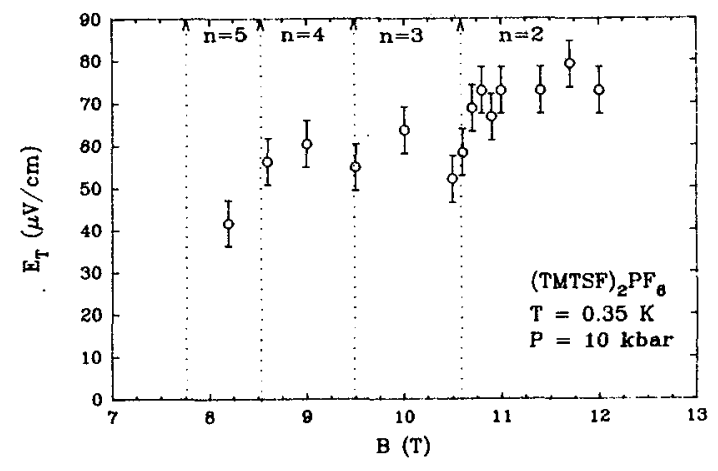

Fig. 4

Figure 3: Temperature dependence of the threshold field $E_{T}$. The solid line is a guide to the eye. Arrow indicates the temperature of the transition to the metallic phase.

Figure 4: Magnetic field dependence of the threshold field $E_{T}$. The pointed lines are the boundaries of the FISDW phases with index $n$.

FISDW. An $E_{T}$ increasing with temperature is again in agreement with the theory [8] and also with the experimental results [9] for SDW's. The roughly factor-of-two increase of $E_{T}$ between $8 \mathrm{~T}$ and $12 \mathrm{~T}$ (see Fig. 4) is also easily understood in this framework, as the FISDW transition temperature and order parameter increase with increasing magnetic field [2]. In conclusion, the existence of a sharp threshold field with properties in good agreement with expectations for FISDW depinning is a strong evidence that the observed nonlinear transport phenomena arise from the depinning and sliding of the FISDW.

The straightforward interpretation of the decrease of $\rho_{x x}$ above $E_{T}$ is that the depinned FISDW carries a charge along the conducting chains, just like in the case of ordinary SDW's or charge-density waves. In a two-fluid model the current is separated to the contributions of the electrons condensed into the FISDW, $I_{c}$, and of the normal electrons, $I_{n}$. The longitudinal and transverse voltages are expressed as

$$
\begin{gathered}
V_{l}=R_{n} I_{n}, \\
V_{H}=R_{H} I_{n},
\end{gathered}
$$

where the latter equation expresses the fact that no FISDW charge transport occurs perpendicular to the 1D chains. From Eqs. (1) and (2), for the differential resistances one has

$$
\frac{d V_{H}}{d I}=R_{H} \frac{d I_{n}}{d I}=\frac{R_{H}}{R_{n}} \frac{d V_{l}}{d I}
$$

Therefore, if the resistivity tensor of the normal carriers $\left(R_{n}\right.$ and $\left.R_{H}\right)$ is not influenced by the depinning of the FISDW $d V_{H} / d I \propto d V_{l} / d I$ should hold. Figure 1 clearly demonstrates that this is not the case: the nonlinearity of $d V_{H} / d I$ is much stronger than that of $d V_{l} / d I$.

This situation is qualitatively similar to what is observed in other SDW [10] or CDW [11] systems, and can be interpreted in an interacting two-fluid model in which Eqs. (1) or (2) break down. In the FISDW states the deviation of $R_{H}$ in Eq. (2) from the quantized value is not expected, and the interaction between the two fluids should appear in Eq. (1). According to the kinetic theory of density wave transport developed by Artemenko and Kruglov [12], the sliding density wave creates a backflow $-\alpha I_{c}$ of normal carriers and (1) is replaced by

$$
V_{l}=R_{n}\left(I_{n}+\alpha I_{c}\right)
$$


where $\alpha$ is a constant independent of electric field. From a comparison of the $d V_{l} / d I$ and $d V_{H} / d I$ curves (Fig. 2) the backflow coefficient $\alpha$ can be inferred. At $T=350 \mathrm{mK}$ we obtain $\alpha=0.8 \pm 0.1$, with no systematic variation with magnetic field between 8 and $12 \mathrm{~T}$. These are the highest values ever reported in any density wave system. The coefficient is close to 1 where the nonlinearity in $\rho_{x x}$ disappears because any charge carried by the FISDW is compensated by a normal carrier backflow of the same magnitude. As discussed elsewhere in this volume [13], the mechanism proposed in Ref. [12] cannot account for such a large $\alpha$.

We note that for $\alpha>1$ (which does not violate any law of thermodynamics) $\rho_{x x}$ increases with electric field as found by Osada et al. [4] in (TMTSF) ${ }_{2} \mathrm{ClO}_{4}$. In this case, however, $\rho_{x y}$ should be strongly nonlinear, which was not observed in the experiments of Ref. [4]. A comparative study of the nonlinear conduction in (TMTSF $)_{2} \mathrm{PF}_{6}$ and (TMTSF) ${ }_{2} \mathrm{ClO}_{4}$ may help to understand the anomalous phase diagram [14] of the latter material.

In conclusion, we have measured nonlinear resistivity along the conducting chains together with a nonlinear Hall resistivity in the FISDW states above a well-defined threshold field $E_{T}$. The existence of a sharp threshold field, as well as the magnitude, temperature, and magnetic field dependence of $E_{T}$ strongly suggest that the origin of the nonlinearity is the depinning of the FISDW. We interpret the nonlinear conduction on an interacting two-fluid model and find an unusually high interaction coefficient the origin of which remains to be further investigated.

We are grateful to C. Lenoir and P. Batail for providing the samples.

\section{References}

[a] On leave from the Institute of Physics, University of Zagreb, Zagreb, Croatia.

[b] Also at Research Institute for Solid State Physics, P.O.Box 49, H-1525 Budapest, Hungary.

[1] J. R. Cooper et al., Phys. Rev. Lett. 63, 1984 (1989); S. T. Hannahs et al., ibid. p.1988.

[2] For a review and further references, see, e.g., G. Montambaux, Physica Scripta T35, 188 (1991).

[3] T. G. Petrova and A. Rozhavsky, this conference; V. M. Yakovenko ibid.

[4] T. Osada, N. Miura, I. Oguro, and G. Saito, Phys. Rev. Lett. 58,1563 (1987).

[5] W. Kang, J. R. Cooper, and D. Jérome, Synth. Metals 41-43, 2083 (1991).

[6] H. Fukuyama and P. A. Lee, Phys. Rev. B 17, 553 (1977);P. A. Lee and T. M. Rice, ibid. 19, 3970 (1978).

[7] G. Mihály and P. Canfield, Phys. Rev. Lett. 64, 459 (1990).

[8] K. Maki and A. Virosztek, Phys. Rev. B 42, 655 (1990).

[9] S. Tomić et al., J. Phys. I (France) 1, 16033 (1991).

[10] O. Trætteberg, L. Balicas, and G. Kriza, this conference.

[11] Yu. I. Latyshev, Ya. S. Savitskaya, V. V. Frolov, Lecture Notes in Physics 217, 339 (1985).

[12] S. N. Artemenko and A. N. Kruglov, Fiz. Tverd. Tela 26, 2391 (1984).

[13] G. Kriza and O. Trætteberg, this conference.

[14] W. Kang, S. T. Hannahs, and P. M. Chaikin, Phys. Rev. Lett. 70, 3091 (1993). 